\title{
Public Health Infos
}

https://doi.org/10.1515/pubhef-2021-0044

\section{„Tagungen“}

\section{6th annual meeting and 11th conference of HEPA Europe}

\author{
„An ecosystem approach to health-enhancing physical \\ activity promotion" \\ 31 August - 2 September 2022 \\ Université Côte d’Azur, Nizza (Frankreich)
}

Veransalter: HEPA Europe (European network for the promotion of health-enhancing physical activity), Université Côte d'Azur, WHO/Europa, Société Française de Santé Publique, Stadt Nizza

In diesem Jahr konzentriert sich die HEPA Europe-Konferenz auf den Schwerpunkt „Ein Ökosystemansatz zur Förderung gesundheitsfördernder körperlicher Aktivität“. Die Konferenzthemen umfassen Politik, Verkehr, Sport, Determinanten von körperlicher Aktivität, Gesundheit und Bewegungsmangel. Ziel ist es, dass sich politische Entscheidungsträger, Fachleute, Wissenschaftler und andere Interessengruppen auf dem Gebiet der Förderung gesundheitsfördernder körperlicher Aktivität engagieren. Die Konferenz bietet Gelegenheit, diese Zielgruppen anzusprechen und $\mathrm{zu}$ verbinden. Nähere Informationen sind auf der Webseite https://hepaeurope2022.sciencesconf. org zusammengesellt.

\section{World Congress of Epidemiology 2021}

\author{
„Methodological Innovations in Epidemiology“ \\ 03.-06.09.2021 \\ Melbourne (Australien)
}

Veranstalter: International Epidemiology Association, Australasian Epidemiology Association

Der Weltkongress für Epidemiologie, Biostatistik und öffentliche Gesundheit findet alle drei Jahre statt und wurde um ein Jahr auf 2021 verschoben. Das übergeordnete Thema des Kongresses lautet „Methodologische Innovationen in der Epidemiologie“, die den innovativen
Fortschritt in den Bereichen Epidemiologie und öffentliche Gesundheit widerspiegeln. $\mathrm{Zu}$ den Unterthemen gehören: Lehren und Kommunizieren von Epidemiologie und Biostatistik, Übersetzung von Forschung in Politik und Praxis sowie Karriereentwicklung für Epidemiologen der frühen bis mittleren Karriere. Eine spezielle Sitzung für COVID-19-bezogene Präsentationen auf dem Kongress soll einbezogen werden. Die Webseite https://wce2020. org/ stellt weitere Informationen zur Verfügung.

\section{Gemeinsamer Kongress DGMP/DGMS 2020}

\section{„Psychosoziale Medizin in Zeiten des Umbruchs“}

16.-17.09.2021

Universitätsklinikum Hamburg-Eppendorf

Veranstalter: Deutsche Gesellschaft für Medizinische Psychologie (DGMP) und Deutsche Gesellschaft für Medizinischen Soziologie (DGMS)

Aktuell erleben wir starke Veränderungen, die sowohl die Welt, unsere Gesellschaft als auch die Fächer der Deutschen Gesellschaft für Medizinische Psychologie (DGMP) und der Deutschen Gesellschaft für Medizinische Soziologie (DGMS) vor Herausforderungen stellen: Dies gilt für die Folgen des globalen Klimawandels, für die aktuelle COVID-19-Pandemie und auch für die Reform des Medizinstudiums und der Approbationsordnung. Für die beiden Fachgesellschaften ist es vor diesem Hintergrund wichtig, eng zu kooperieren und sich gemeinsam zu positionieren. Neben diesen Herausforderungen werden weitere Themen auf dem Kongress eine Rolle spielen: Patientenzentrierte Medizin, Kommunikation in der Medizin, Public Mental Health und Mental Health Care. Auf der Webseite http://dgmp-dgms-2021.de/ finden sich Informationen zum Kongress.

\section{Jahrestagung der DGEpi}

\section{„Krankheiten erforschen -Gesundheit erhalten“}

20.-22.09.2021

Würzburg

Veranstalter: Deutsche Gesellschaft für Epidemiologie e.V. (DGEpi), Institut für Klinische Epidemiologie und Biometrie (IKE-B) 
Die Deutsche Gesellschaft für Epidemiologie (DGEpi) trifft sich im September 2021 unter dem Motto „Krankheiten erforschen - Gesundheit erhalten“ zur 16. Jahrestagung in Würzburg. Die Erkenntnisse aus den Studien der Gesellschaft dienen dazu, Gesundheitsvorsorge und -versorgung $\mathrm{zu}$ erneuern und zu optimieren sowie Entscheidungsprozesse im sozial- und gesundheitspolitischen Bereich mit validen Daten zu unterstützen. Weitere Informationen zur Veranstaltung und Registrierung werden auf der Webseite www.dgepi. de/de/tagungen-and-fortbildungen zur Verfügung gestellt.

\section{Deutsche Gesellschaft für Sozialmedizin und Prävention (DGSMP)}

\section{Jahrestagung der DGSMP 2020 in Leipzig}

\section{„Das Soziale in Medizin und Gesellschaft - Aktuelle Megatrends fordern uns heraus“}

\section{2.-24.09.2021}

Medizinische Fakultät der Universität Leipzig

Veranstalter: Deutsche Gesellschaft für Sozialmedizin und Prävention e.V. (DGSMP)

Die ursprünglich in 2020 geplante 56. Jahrestagung der DGSMP wird im September 2021 in Leipzig stattfinden. Das Thema der Tagung bleibt „Das Soziale in Medizin und Gesellschaft - Aktuelle Megatrends fordern uns heraus“. Megatrends, wie Globalisierung, Individualisierung, Urbanisierung, Digitalisierung und Global Aging, sind gesellschaftliche Entwicklungen, die langfristig und tiefgreifend unsere sozialen, politischen und ökonomischen Verhältnisse verändern. Megatrends beeinflussen uns, unser soziales Miteinander, unsere Gesundheit und die Gesundheitsversorgung der Bevölkerung in vielfältiger Weise. Die Tagung lotet Chancen und Barrieren dieser Entwicklungen aus. Es werden aktuelle Aspekte, wie u.a. Chancen von E-Health-Interventionen, Herausforderungen bei der Versorgung von Flüchtlingen, Prävention von Demenzen oder Sicherstellung der Gesundheitsversorgung auf dem Lande aufgegriffen. Informationen zur Tagung und Anmeldung sind auf der Seite www. dgsmp2021-leipzig.de zusammengestellt.

\section{„Related Links“}

Das Bundesministerium für Familie, Senioren, Frauen und Jugend leitet den Nutzer auf der Webseite www. bmfsfj.de über das Thema Familie mit dem Unterthema Kinderwunsch und Schwangerschaft hin zu Pränataldiagnostik (https://www.bmfsfj.de/bmfsfj/themen/familie/ schwangerschaft-und-kinderwunsch/praenataldiagnostik). Die Webseite fasst Informationen zum Thema Pränataldiagnostik und $\mathrm{zu}$ Beratungsmöglichkeiten bei auffälligem Befund zusammen und verlinkt zu weiterführenden Materialien, Publikationen und Links.

Gesundheitsziele.de ist ein Kooperationsverbund zur Weiterentwicklung des nationalen Gesundheitszieleprozesses. Unter Beteiligung von Bund, Ländern und Akteuren des Gesundheitswesens werden Gesundheitsziele entwickelt und Maßnahmen zur Zielerreichung empfohlen. $\mathrm{Zu}$ den bisher formulierten Zielen gehört auch "Gesundheit rund um die Geburt“. Auf der Seite www. gesundheitsziele.de werden unter dem Reiter „Nationale Gesundheitsziele“ jeweils Status quo, Relevanz, das Ziel selbst und empfohlene Startermaßnahmen zum Gesundheitsziel zusammengefasst.

Die Informations- und Vernetzungsstellen Pränataldiagnostik (IuV-Stellen PND) in Baden-Württemberg sind ein vom Landesministerium für Arbeit und Sozialordnung, Familien und Senioren gefördertes Projekt zum Aufbau eines breiten interprofessionellen Netzwerks. Die Webseite www.pnd-beratung.de informiert über Anlässe für vorgeburtliche Untersuchungen, invasive und nicht-invasive Untersuchungen und deren Möglichkeiten und Grenzen. Erläutert werden die rechtlichen Grundlagen vorgeburtlicher Untersuchungen sowie das Beratungsangebot unterschiedlicher Professionen zu vorgeburtlichen Untersuchungen. Des Weiteren gibt es einen Bereich mit aktuellen Meldungen und mit FAQs. Abgerundet wird dieses regional unabhängige Informationsangebot durch Verlinkung zu Adressen und Terminen in Baden-Württemberg.

Die Webseite www.familienplanung.de der Bundeszentrale für gesundheitliche Aufklärung richtet sich an werdende Eltern und begleitet den Zeitraum vom Kinderwunsch über Schwangerschaft, Geburt und die erste Zeit mit Kind. Im Themenbereich Schwangerschaft informiert die Seite über den Schwangerschaftsverlauf, die kindliche Entwicklung während der Schwangerschaft, Schwangerschaftsvorsorge und Pränataldiagnostik. Unter dem Reiter Beratung \& Hilfen werden der Anspruch auf Beratung sowie Beratungsthemen, -arten und -stellen erläutert. Zusätzlich werden Anregungen für Gesundheit und Ernährung und Hilfestellung für Organisatorisches und finanzielle Fragen zur Verfügung gestellt. 
Frühe Hilfen sind Angebote für Eltern ab der Schwangerschaft und Familien mit Kindern bis drei Jahre. Diese Angebote kommen aus verschiedenen Systemen, insbesondere aus dem Bereich der Kinder- und Jugendhilfe, dem Gesundheitswesen, der Frühförderung und der Schwangerschaftsberatung. Fachkräfte dieser Bereiche arbeiten eng zusammen, um Eltern bei der Betreuung und Förderung ihrer Kinder zu unterstützen. Sie werden in lokalen Netzwerken koordiniert. Das Nationale Zentrum Frühe Hilfen (NZFH) ist damit betraut, die Weiterentwicklung der Frühen Hilfen zu unterstützen. Dies geschieht unter anderem durch wissenschaftliche Forschung, durch Angebote zur Qualitätsentwicklung, durch Fachaustausch mit Akteuren der Frühen Hilfen aus Bund, Ländern und Kommunen sowie durch verfügbar gemachte Materialien zur Fort- und Weiterbildung der Fachkräfte in den Frühen Hilfen. Die Seite www.fruehehilfen.de bietet Hintergründe und rechtliche Rahmenbedingungen sowie umfassende Informationen für unterschiedliche Akteure und $\mathrm{zu}$ Fachthemen der Frühen Hilfen. Es gibt Verlinkungen zu Landeskoordinierungsstellen, Kontaktdaten, gesetzliche Regelungen und Konzepte der Länder. Akteuren in den Frühen Hilfen werden Fachinformationen und Praxismaterial zur Verfügung gestellt.

\section{„Weiterführende Literatur“}

Robert Koch-Institut. Gesundheitsberichterstattung des Bundes. Gesundheit in Deutschland [Health Report of the Federal State. Health in Germany.]. Robert Koch-Institut (Hrsg) (2015) Gesundheit in Deutschland. Gesundheitsberichterstattung des Bundes. Gemeinsam getragen von RKI und Destatis. RKI, Berlin. Berlin: Robert-Koch-Institut; 2015.

Scholaske L, Spallek J, Entringer S. Intergenerationale Übertragung von Migrationserfahrungen und fetale Programmierung von Krankheit und Gesundheit. In: Spallek J, Zeeb H, editors. Migration und Gesundheit. Bern: Hogrefe.

Kuh D, Shlomo YB. A life course approach to chronic disease epidemiology: conceptual models, empirical challenges and interdisciplinary perspectives. Int J Epidemiol 2002;31:285-93.

Wolff J, Graumann S. Aktueller Stand und Entwicklungen von Pränataldiagnostik: Gutachten im Auftrag des Deutschen Bundestages, vorgelegt dem Büro für Technikfolgen-Abschätzung beim Deutschen Bundestag (TAB). Bochum: Evange-lische Hochschule Rheinland-Westfalen-Lippe; 2016.

Beauchamp TL, Childress JF. Principles of biomedical ethics. 6th Edition. ed: Oxford University Press; 2008.

Cignacco E, Berger A, Sénac C, Wyssmüller D, Hurni A, zu SaynWittgenstein F. Sexuelle und reproduktive Gesundheitsversorgung von Frauen und ihren Säuglingen in Asylunterkünften in der Schweiz (REFUGEE). Eine Situationsanalyse und Empfehlungen.
Bern: Berner Fachhochschule, Departement Gesundheit, Fachbereich Geburtshilfe; 2017.

Wieners K, Hellbernd H, Jenner SC, Oesterhelweg L. Häusliche Gewalt in Paarbeziehungen - Intervention und Prävention. Notfall Rettungsmed 2012, 15:65-80 DOI 10.1007/s10049-011-1559-0 @ Heidelberg: Springer-Verlag; 2012.

Bundesministerium für Gesundheit. Herausgeber. Nationales Gesundheitsziel Gesundheit rund um die Geburt. Berlin: BMG, 2017.

Kälble, K. Borgetto, B. Soziologie der Berufe im Gesundheitswesen. In: Richter, M. Hurrelmann, K. Hrsg. Soziologie von Gesundheit und Krankheit. Wiesbaden. Springer VS, 2016:383-402.

Seichter, S. Erziehung an der Mutterbrust. Eine kritische Kulturgeschichte des Stillens. Weinheim, Basel: Juventa, 2014.

Kröger, M, Rückert-John, J. Stillende‘ Männer. Väterselbstbilder und Väterfremdbilder im Übergang zur Elternschaft. In: Seehaus, R, Rose, L, Günther, M, Hrsg. Mutter, Vater, Kind - Geschlechterpraxen in der Elternschaft. Opladen. Budrich, 2015:81-99.

Rose, L Planitz B. Der ungleiche Start ins Leben. Soziale Differenzen ,rund um die Geburt' als wissenschaftliche und sozialpolitische Herausforderung. In: Mitscherlich-Schönherr 0 , Anselm R Hrsg. Gelingende Geburt. Interdisziplinäre Erkundungen in umstrittenen Terrains. Berlin, Boston. De Gruyther, 2021:247-69, $255 f$.

Wall, G. Moral Construction of Motherhood in Breastfeeding Discourse. Gender \& Society 2001;4:592-610. Kröger M, Rückert-John, J. Stillen als Quelle von Gesundheit und Glück. Die Rekonstruktion traditioneller Geschlechterrollen durch natürliche Mütterlichkeit. In: Hoefert, H, Klotter, C. Hrsg. Gesundheitszwänge. Lengerich. Pabst, 2013:189-206.

Flaake 2014. Rüling, A. Jenseits der Traditionalisierungsfallen. Wie Eltern sich Familien- und Erwerbsarbeit teilen. Frankfurt a.M.: Campus Verlag, 2007.

Rose, L, Pape, J. Geschlechterordnungen der Familie. Ethnografische Eindrücke zur Positio-nierung des Vaters in Bildungsangeboten zur Geburt und ersten Lebenszeit des Kindes. In: Rose, L, Schimpf, E, Hrsg. Sozialarbeitswissenschaftliche Geschlechterforschung. Opladen. Budrich, 2020:183-200.

Pape, J Von Brust zu Brei - Maternalisierung von Ernährungsverantwortung in Beikostkursen. In: Tolasch, E, Seehaus, R, Hrsg. Mutterschaften sichtbar machen. Sozial- und kulturwissenschaftliche Beiträge. Opladen. Budrich, 2015:255-70.

Werdecker L, Esch T. Bedeutung und Rolle von Gesundheitsberufen in der Prävention und Gesundheitsförderung. Heidelberg. Prävention und Gesundheitsförderung Springer; 2019.

Schäfers R. Gesundheitsförderung durch Hebammen. Fürsorge und Prävention rund um Mutterschaft und Geburt. Stuttgart: Schattauer; 2011.

Bauer NH. Das Versorgungskonzept Hebammenkreißsaal und die möglichen Auswirkungen auf Gesundheit und Wohlbefinden von Mutter und Kind. Göttingen: V \& R unipress; 2011.

Luksch K, Villmar A, Bauer NH. Hebammenzentralen-Schlüsselfaktor zur Arbeitserleichterung von Hebammen? Deutsche Gesellschaft für Hebammenwissenschaft 5 Internationale Konferenz der Deutschen Gesellschaft für Hebammenwissenschaft (DGHWi) Bochum, 13-14022020 Düsseldorf: German Medical Science GMS Publishing House. 2020. 


\section{„Lehre“}

\section{Die Modellstudiengänge für Therapieberufe haben sich bewährt - jetzt müss(t)en die Berufsgesetze novelliert werden}

Seit 10 Jahren werden aufgrund von Modellklauseln in den Berufsgesetzen primärqualifizierende Studiengänge in Physiotherapie, Ergotherapie und Logopädie erprobt und evaluiert.

Das Bundesministerium für Gesundheit plant nun eine Verlängerung der Modellklauseln um weitere fünf Jahre (bis 2026; s. Referentenentwurf zum Gesundheitsversorgungsweiterentwicklungsgesetz - GVWG vom Oktober 2020).

Der Hochschulverbund Gesundheitsfachberufe (HVG) befürchtet, dass durch diese weitere - sachlich völlig unbegründete - Verlängerung der begonnene Akademisierungsprozess und die Reform der Berufsgesetze auf die lange Bank geschoben werden. Wenn dies nicht das Ziel der Politik ist, dann müssen das Studium als regulärer Ausbildungsweg für die Therapieberufe anerkannt und in den Berufsgesetzen verankert werden. Das BMG scheint das nicht mehr in dieser Legislaturperiode umsetzen zu wollen oder zu können, so die Vermutung des Hochschulverbundes Gesundheitsfachberufe (HVG). Der HVG setzt sich - gemeinsam mit den großen Berufsverbänden der Ergo- und Physiotherapie sowie der Logopädie - für eine unverzügliche Ausbildungsreform und eine vollständig hochschulische Berufsausbildung der drei Therapieberufe ein; seine Stellungnahme zu dem GVWG-Entwurf findet sich unter www.hv-gesundheitsfachberufe.de

Kontakt: Prof. Dr. Jutta Räbiger, Vorstandsmitglied des HVG 